\title{
Diet quality and nutritional adequacy of young children in the UK according to their consumption of young child formula and commercial infant food
}

\author{
E.O. Verger ${ }^{1,2}$, S. Eussen ${ }^{3}$ and B.A. Holmes ${ }^{4}$ \\ ${ }^{1}$ Institute of Cardiometabolism and Nutrition (ICAN), Pitié-Salpêtrière Hospital, Paris, France, ${ }^{2}$ Institut National de \\ la Santé et de la Recherche Médicale (INSERM), Research Unit S1166 (UMR S 1166), Paris, France, ${ }^{3}$ Danone \\ Nutricia Research, Utrecht, The Netherlands and ${ }^{4}$ Danone Nutricia Research, Palaiseau, France
}

It is well recognized that the first two years of life are particularly important for later life health, and adequate nutrition is essential to optimize health, physical and mental development, and reduce the risk of chronic disease ${ }^{(1)}$. Between 6 and 24 months of age, children are switching from an exclusive milk diet to more adult-like meals and snack patterns which involve an increased variety of foods and nutrient compositions ${ }^{(2)}$. The potential contribution that Young Child Formula (YCF) and Commercial Infant Food (CIF) make to a healthy diet of children aged 12 months and over remains unclear. Diet quality indexes are useful tools to provide an overall measure of the quality of the diet based on current nutrition knowledge ${ }^{(3)}$. The PANDiet, a nutrient based diet quality index, has been developed and previously validated on adult populations ${ }^{(4)}$. Since the PANDiet measures diet quality in terms of global nutrient adequacy rather than food intakes, it can be used to elucidate the contribution that different food groups make to the nutrient adequacy of the diet. The objectives of this study were to adapt and validate the PANDiet for UK young children aged 12 to 18 months and determine the nutritional adequacy of the diets in groups of children according to their YCF and CIF consumption. Secondary analysis was undertaken on 1152 young children aged 12-18 months from the UK Diet and Nutrition Survey of Infants and Young Children $\left(\right.$ DNSIYC, 2011) ${ }^{(5)}$

The PANDiet was adapted to the UK based on 25 nutrients, using the UK and EFSA nutritional recommendations for children aged 12 to 36 months. Validity of the PANDiet was assessed by studying associations between the PANDiet and its components, energy intake, food intakes and child and maternal characteristics. Four groups of children were defined according to their intake of YCF and CIF: no consumption, consumption of YCF, consumption of CIF, consumption of YCF and CIF. PANDiet scores and food intakes of these four groups were compared.The validation indicated a lower PANDiet score was linked with lower intakes of $\mathrm{YCF}, \mathrm{CIF}$, vegetables and fruits. Determinants of having a lower score were being older, having siblings and having a younger mother with a lower educational level. Compared to children consuming neither YCF nor CIF, the PANDiet scores were higher in children consuming CIF (+1.4), children consuming YCF $(+7 \cdot 2)$ and children consuming YCF and CIF $(+7 \cdot 8$, all $P<0 \cdot 001)$. More specifically, the probability of adequacy for SFA, PUFA, vitamin D, zinc, iron and copper were higher in children consuming YCF and children consuming YCF and CIF (all $P<0 \cdot 001$ ). Some differences in dietary patterns were seen across the four groups but intakes of vegetables, fruits, fish nor water intakes differed.

These results indicate that the PANDiet is a valid indicator of the nutrient adequacy of the diet of UK young children. Consuming CIF was not found to be associated to a lower nutritional adequacy whereas consuming YCF was associated to a higher nutritional adequacy. Further analysis with greater numbers of subjects is needed to better understand the nutritional adequacy of the diet of different levels of consumption of YCF and CIF in this and other populations.

This study was supported by Danone Nutricia Research.

1. WHO (2014) Infant and young child feeding. Fact sheet $\mathrm{N}^{\circ} 342$

2. Skinner JD, Ziegler P, Pac S et al. (2004) J Am Diet Assoc 104, Suppl. 1, S65-S70.

3. Waijers PMCM, Feskens EJM, Ocké MC (2007) Br J Nutr 97, 219-231.

4. Verger EO, Mariotti F, Holmes BA et al. (2012). PloS One 7, e42155.

5. Lennox A, Sommerville J, Ong K et al. (2013) Diet and nutrition survey of infants and young children, 2011. A survey carried out on behalf of the Department of Health and Food Standards Agency. London, UK. 